\title{
Modeling the detection of optical sights using retro-reflection
}

\author{
Arjan L. Mieremet ${ }^{\mathrm{a}}$, Ric (H.)M.A. Schleijpen ${ }^{\mathrm{a}}$, P.N. Pouchelle ${ }^{\mathrm{b}}$ \\ ${ }^{a}$ TNO Defense, Security and Safety, P.O. Box 96864, 2509 JG The Hague, The Netherlands \\ ${ }^{\mathrm{b}}$ ENSIETA, 29806 Brest Cedex 9, France
}

\begin{abstract}
Retro-reflection can be used for the detection and classification of optical systems. The probability of detecting sights over large ranges depends on parameters of the laser, the sight, the detector and the atmosphere. We have developed a software tool that simulates a sight detection system. With the use of this tool we can 'test' different sight detection system designs and make estimations on detection ranges of optical systems. In this paper we give a short overview of the physical aspects that have been implemented in the model and discuss the experimental validation of our model.
\end{abstract}

Keywords: Detection, retro-reflection, lasers

\section{INTRODUCTION}

The detection of cameras or other optical devices in the background could help military forces to detect a possible attack. The sooner a threat is detected the better, and therefore a method that can detect a threat before being activated is favored. Since a wide range of threats exists, such as snipers and anti-tank weapons, the detection method must be generic. Fortunately, most weapon systems have a common feature: an optical sight. By scanning the surroundings with a laser beam, a relatively strong retro-reflection signal is created by an optical sight that is pointed in the direction of the laser source. This effect is also known as the cat's eye effect.

Currently, detection of retro-reflected signals is used for sniper detection, while it is expected that it can be used in future Directed InfraRed CounterMeasure (DIRCM) systems for fine-tracking of seekers and in closed-loop DIRCM systems for seeker identification.

Retro-reflection techniques are also considered for communication with UAV's and for Identification Friend or Foe (IFF) techniques. In these applications the retro-reflected signal is modulated by a corner cube to transfer information.

To estimate the capabilities of detection by retro-reflection it would be useful to have a simple formula that estimates the amount of power that is returned. However, as will become clear in this paper, the strength of the retro-reflected signal depends on numerous parameters implying that a simple estimation formula is not possible. Therefore we have developed a MATLAB ${ }^{\circledR}$ model that takes into account the major effects that influence the strength of the retro-reflected signal. This model can be used to investigate the range over which optical devices can be detected.

This paper is organized as follows; in section 2 we start with a description of the physical processes that are to be taken into account for correctly calculating the strength of a retro-reflected signal. In section 3 we show some screenshots of the tool that has been developed and in section 4 the results of one of our validation experiments are shown.

\section{THE CAT'S EYE EFFECT}

With the use of Figure 1 we will shortly explain the principle of the cat's eye effect. A parallel beam of radiation enters the eye and is focused onto a single point on the retina (the image plane). The position of this point is related to the direction of incidence of the beam. Each direction of incidence corresponds with one point on the retina. Due to the principle of reversibility of rays the opposite is also the case, namely each point on the retina corresponds with one direction of incidence. Not all radiation is absorbed by the retina, so that a part is either reflected or scattered back towards the lens. The radiation is collected by the lens and send into the direction where the original source of the radiation was located.

Laser Radar Technology and Applications XIII, edited by Monte D. Turner, Gary W. Kamerman Proc. of SPIE Vol. 6950, 69500E, (2008) · 0277-786X/08/\$18 - doi: 10.1117/12.774634 


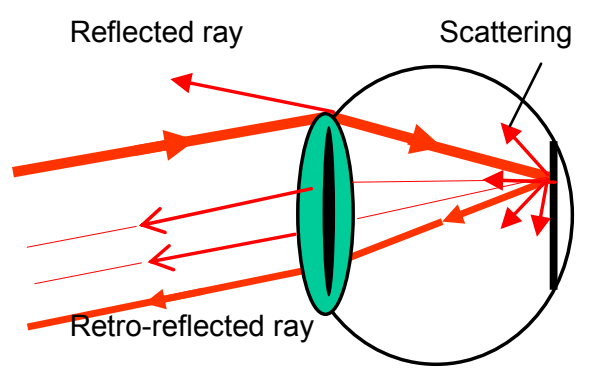

Figure 1: The cat's eye effect.

Seen from the position of the source of the IR beam, radiation that retro-reflects in an optical sight could be orders of magnitude stronger compared to radiation that scatters at some other surface due to the collecting power of the lens. For this reason the cat's eye effect can be used for the detection of optical sights. It is interesting to know which parameters determine the distance over which this effect can be used for detection.

In what follows, we discuss, to our opinion, the five major effects that influence the intensity of the retro-reflected beam and which have been implemented in our model.

\section{Beam spreading}

The intensity of the retro-reflected beam depends on the distance between laser and optical sight. In radar, this dependence is known to be proportional with $R^{4}$ with $R$ the distance between emitter and object. The fact that the exponential is equal to 4 comes from the fact that both the emitted signal and reflected signal are spherical waves. At optical wavelengths this might not always be the case. Let's consider a collimated circular laser beam with waist $w_{0}$ and wavelength $\lambda$ propagating over a certain distance. Initially the waist of this beam will remain constant, i.e. $w_{0}$. This regime is known as near-field. When the propagated distance becomes (much) larger than $\sim \pi w_{0}{ }^{2} / \lambda$, the laser beam starts to diverge. This is known as beam spreading and means that we are in the so-called far-field of the laser. In the nearfield regime the intensity of the laser beam is proportional with $R^{0}$, while in the far-field it goes with $R^{2}$.

The same effect also occurs with the retro-reflected beam. However, the diameter of this beam is largely determined by the size of the entrance pupil of the sight and not by the original beam waist. Therefore the beam spreading angle might be considerably different implying that the distance dependence is not automatically the same as for the beam that propagated towards the optical sight. The intensity of the retro-reflected beam at the receiver is therefore proportional with $R^{\text {a }}$ with $a$ being a non-integer value between 0 and 4 .

So far we considered only collimated beams. However, as we will see later, it is very likely that the retro-reflected beam will be divergent or convergent, in which case beam spreading can be neglected. Whether the beam is collimated or not, the total power of the retro-reflected beam depends on the size of the beam at the optical sight and at the detector. To include all possible cases, the following expression for the beam waist $w$ of a laser beam with a Gaussian intensity profile at a distance $R$ is used (Ref. 1)

$$
w(R)=w_{0} \sqrt{\left(1-R / F_{0}\right)^{2}+\left(\lambda R / \pi w_{0}^{2}\right)^{2}},
$$

where $F_{0}$ is the radius of curvature of the beam at the exit of the laser. The value of $F_{0}$ determines whether we deal with a collimated $\left(F_{0}=\infty\right)$, divergent $\left(F_{0}<0\right)$ or convergent $\left(F_{0}>0\right)$ beam.

The expression given above is not valid for beams with a top-hat intensity profile. For this case a more extensive calculation which includes the calculation of the complete diffraction pattern of the beam is implemented in the model. 


\section{Vignetting}

A tilted sight will produce a much weaker retro-reflected signal due to vignetting of the pupil. Figure 2 represent a case of vignetting. The dashed lines show the propagation of a beam that is located on the optical axis of the optical sight. An image of the laser is formed in the image plane of the sight. The retro-reflected beam starts at this image plane and propagates back towards the laser. For clarity, we have shown the retro-reflected beam on the right side of the image plane. The lens on the right side is thus the same lens as the one on the left.

The continuous line corresponds to a case where the laser is not located on the optical axis, i.e. the optical sight is tilted. The laser is still sharply imaged on the image plane and it is assumed that the rays specularly reflect at this image plane. The propagation of the reflected beam is shown on the right side of the image plane. The lens shown at that side is thus the same lens as the one shown on the left. From this figure it can be seen that the shape of the retro-reflected beam not matches with the lens, i.e. the beam gets vignetted.

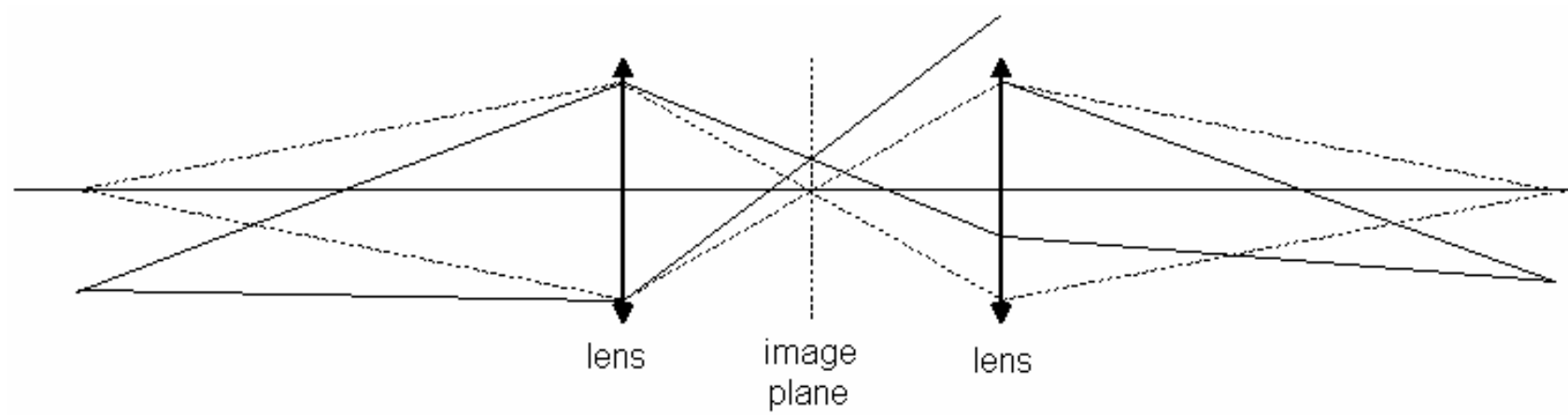

Figure 2: The vignetting effect. When the optical sight is tilted, part of the retro-reflected beam will not exit the sight.

The influence of vignetting must not be underestimated. A tilt of several degrees could already be sufficient to completely block the retro-reflected signal. This implies that the optical sight is pointed into another direction and can be considered to be non-dangerous. However, the other direction could be a location close to the observer, depending on the distance, which might also need to be protected.

In the tool the optical sight is modeled as being a single lens combined with an image plane which specularly reflects a portion of the incoming radiation. The user can control the amount of vignetting by tilting the optical sight.

\section{Defocusing}

Another important factor is the observation distance of the optical sight. When the optical sight is exactly focused on the laser, the retro-reflected beam will also be focused in this plane as can be seen in Figure 2. However, as soon as the optical sight is focused on another distance, the retro-reflected beam will likely be strongly divergent or convergent. In Figure 3 a case is shown where the optical sight is focused onto a point beyond the laser. The dashed line represents rays coming from an object at the observation distance, while the continuous lines represent the laser beam. The laser spot will be imaged behind the image plane and as a consequence the retro-reflected beam is strongly divergent (strongly exaggerated in the figure). 


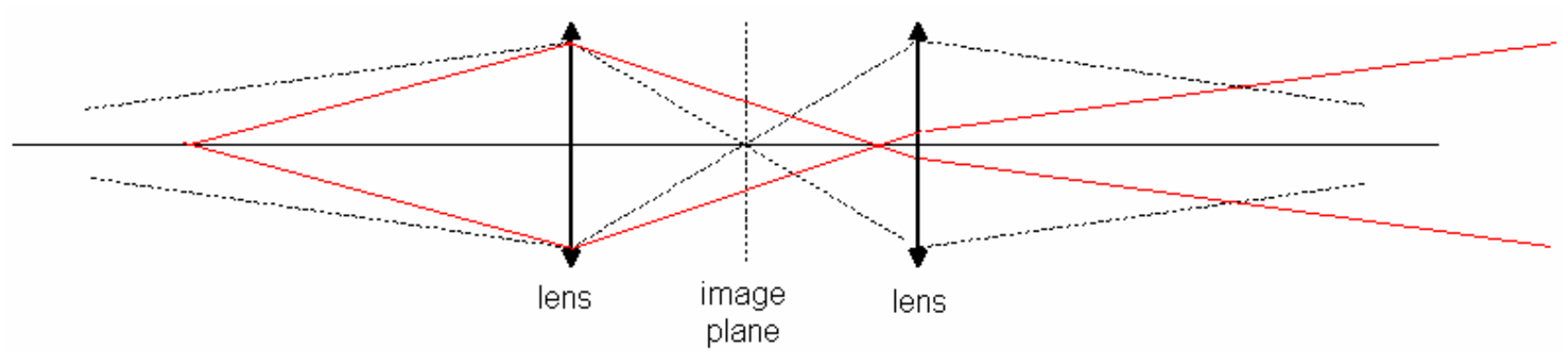

Figure 3: The defocusing effect. When the laser is not located at the distance of observation, the retro-reflected beam might be strongly divergent.

From Figure 3 it can also be seen that the size of the beam exiting the optical sight is different from the size of the beam that entered. Although in the example shown the beam is much smaller, situations exist where the exit beam diameter could also be larger than the aperture implying an additional loss due to vignetting.

This additional loss and the divergence angle of the beam exiting the optical sight are taken into account by the tool. In this way the tool allows the user to investigate how the strength of the retro-reflected signal depends on the observation distance of the optical sight.

\section{Atmospheric transmission}

To model atmospheric transmission, we have used the model based on Koschmieder (Ref [2]).

$T_{\text {atm }}=e^{-\sigma R}$ with $\sigma=\left(\frac{3.912}{R_{v i s}}\right)\left(\frac{0.55}{\lambda}\right)^{0.585 R_{v i s}^{1 / 3}}$

where $R_{\text {vis }}$ is the meteorological range and $\lambda$ the wavelength in microns, which is an internationally defined standard, ranging from 'dense fog' $(0-50 \mathrm{~m})$ to 'exceptionally clear' $(>50 \mathrm{~km})$. Several remarks are to be made when using this expression. First, by using this expression, it is assumed that the transmission is fully determined by scattering at aerosols and that the wavelength is not on a gas absorption line. Second, the expression assumes that the spectral response is that of a human eye, which not the case is since detectors are used in practice. Third, the expression is wavelength dependent, but is limited for wavelengths up to $3 \mu \mathrm{m}$.

Based on the above mentioned remarks it must be concluded that the calculation of the atmospheric transmission in our tool is just a first order approximation.

The user can set the weather type from exceptionally clear up to heavy fog, each corresponding with a specific value of the transmittance.

\section{Atmospheric turbulence}

In the presence of atmospheric turbulence there are two effects that influence the total intensity of the retro-reflected beam. The first effect is that the laser beam undergoes an additional beam spreading effect causing the beam to be larger than expected from diffraction effects only. An expression for this additional effect is (Ref. 1)

$w_{e}=w \sqrt{1+1.63 \sigma_{1}^{12 / 5} \Lambda}$ with $\sigma_{1}^{2}=1.23 C_{n}^{2} k^{7 / 6} R^{11 / 6}$ and $\Lambda=\lambda R / \pi w^{2}$

$C_{\mathrm{n}}^{2}$ is the atmospheric structure constant and $k$ the wavenumber $(2 \pi / \lambda)$.

Second, the central position of the beam changes with time, i.e. also known as beam wandering. As a consequence, the intensity received at the detector varies with time. To model this effect a turbulence spectrum has been assumed. The theoretical spectrum shape for plane waves is $(\operatorname{Ref}[3])$ 
$\operatorname{PSD}(f)=\frac{2.192}{2.577+\left(f / f_{0}\right)^{8 / 3}}\left(\frac{C_{n}^{2}}{v}\right) k^{2 / 3} R^{7 / 3}$ with $f_{0}=0.4 \frac{v}{\sqrt{\lambda / R}}$

where $f_{0}$ is a cutoff frequency determined by the transverse wind speed $v$, wavelength $\lambda$ and propagation distance $R$. The value $8 / 3$ corresponds to the Kolmogorov structure function in two dimensions. For frequencies much smaller than $f_{0}$, the spectrum is flat, while for high frequencies the spectrum goes with $\left(f l f_{0}\right)^{-8 / 3}$. To create a power signal that has this power spectrum, a random phase is assigned to each frequency and then the inverse Fourier transform is taken.

\section{Other effects}

Above, we have described five major effects influencing the strength of the retro-reflected signal. These effects have been incorporated in our model to be able to make a good estimation of the collected laser power. Besides the above mentioned effects, also other effects such as thermal noise from the detector and wavelength filters can be modeled.

Another effect we haven't mentioned is that in its current form, our model assumes that the retro-reflected beam is created by specular reflection. The model would be more accurate if a scattering component is taken into account. This feature will be implemented in a future version of the tool. The user however can control the reflection coefficient of the image plane.

\section{SIMULATION TOOL}

Our model has been divided into four main parts; the source, the optical sight the detector and atmosphere.

The laser is defined by several parameters, such as the power, aperture diameter, wavelength and intensity profile. Based on these parameters the total power on the receiver is determined. After that, the parameters such as pulse pattern and pulse cycle are applied to determine the time dependence of the received signal.

As might have become clear from the previous section, the optical sight is modeled as being a lens and a specular reflecting image plane. Besides the possibility to control the reflection coefficient of the image plane, also the transmission of the lens can be set.

The detector consists of a lens with a given diameter and an avalanche photodiode (APD). It is assumed that all power collected by the lens falls upon the sensitive area of the detector. The user can amongst others provide data on the quantum efficiency of the detector and the temperature of the detector to simulate thermal noise.

In Figure 4 the input screen of the retro-reflection tool is shown. This image shows four blocks corresponding to the laser (top-left), optical sight (top-right), detector (bottom-left) and atmosphere (bottom-right). In the center, several parameters for the visualization of the results can be controlled.

When all parameters are set, the 'launch all' button can be pressed and the tool starts evaluating the created setup. When the tool is finished, a result screen appears (Figure 5). This result window shows the values of the most interesting parameters (left-hand side) and images of the intensity distribution of the beam at different stages are shown (right-hand side). 


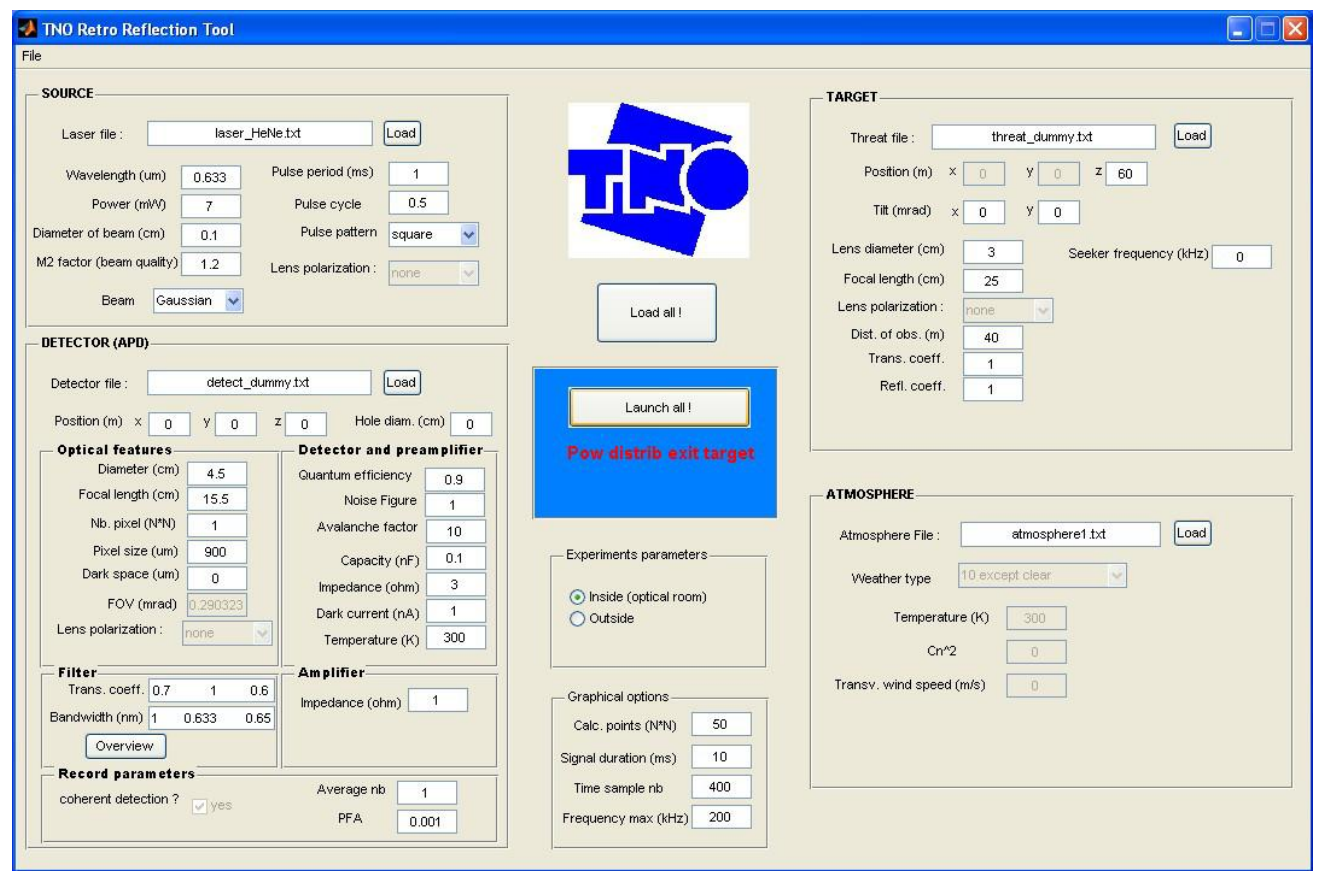

Figure 4: Screenshot of the input of the retroreflection tool. The input is divided into four blocks.

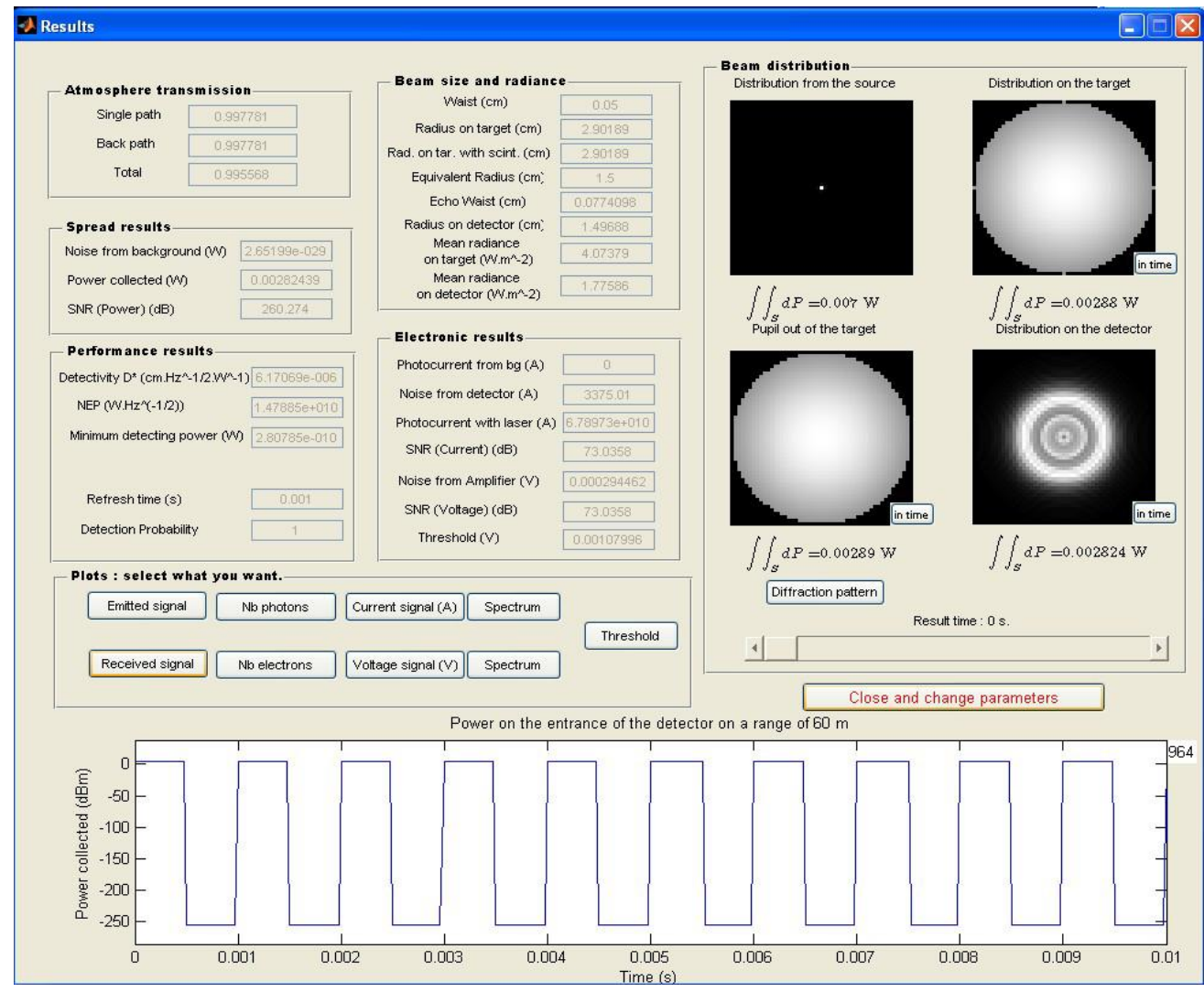

Figure 5: Screenshot of the output of the retroreflection tool. 


\section{MODEL VALIDATION}

To validate the model, simple experiments have been performed to verify that the model correctly predicts the different effects implemented in the tool. In this paper we show the validation of the defocusing effect. First we describe the setup and then show the prediction of our tool and compare it with the measurements.

\subsection{Setup description}

A sight simulator consisting of a lens (diameter $30 \mathrm{~mm}$, focal length $25 \mathrm{~cm}$ ) and a mirror were placed at a distance of roughly $60 \mathrm{~m}$ from a HeNe laser $(\lambda=633 \mathrm{~nm})$. The mirror functioned as a perfect reflector in the focal plane of the lens. During the measurements the mirror is translated along the optical axis out of the focus position over a distance $\varepsilon$ ranging from $-7 \mathrm{~mm}$ to $7 \mathrm{~mm}$. When $\varepsilon=0 \mathrm{~mm}$, the sight simulator is focused on the laser source.

The detector consisting of a lens with a diameter of $30 \mathrm{~mm}$ and its centre was located $75 \mathrm{~mm}$ from the laser. This implies that the edge closest to the laser was at a distance of $60 \mathrm{~mm}$, while the edge at the opposite site was at a distance of $90 \mathrm{~mm}$. All the laser radiation collected by the lens fell upon the sensitive part of the detector.

In Figure 6 a schematic drawing of the setup is shown. The solid lines correspond with the laser beam propagating towards the optical sight, while the dashed lines correspond to the retro-reflected beam. In this figure we show the case where the image plane is located too close to the lens, i.e. $\varepsilon<0$, causing the retro-reflected beam to be divergent. Figure 7 shows a photo of the lens and mirror that have been used to simulate an optical sight.

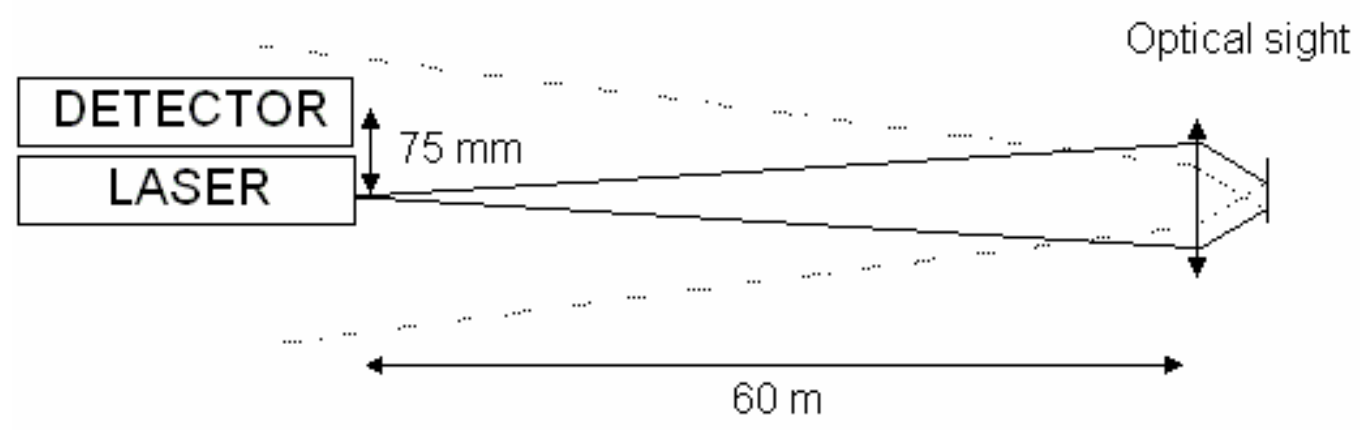

Figure 6: Schematic drawing of the setup. On the left side a laser and detector are located. The laser illuminates the sight simulator that is positioned $60 \mathrm{~m}$ from the laser. Depending on the mirror position with respect to the image plane of the lens, the retro-reflected beam is divergent, collimated or convergent. 


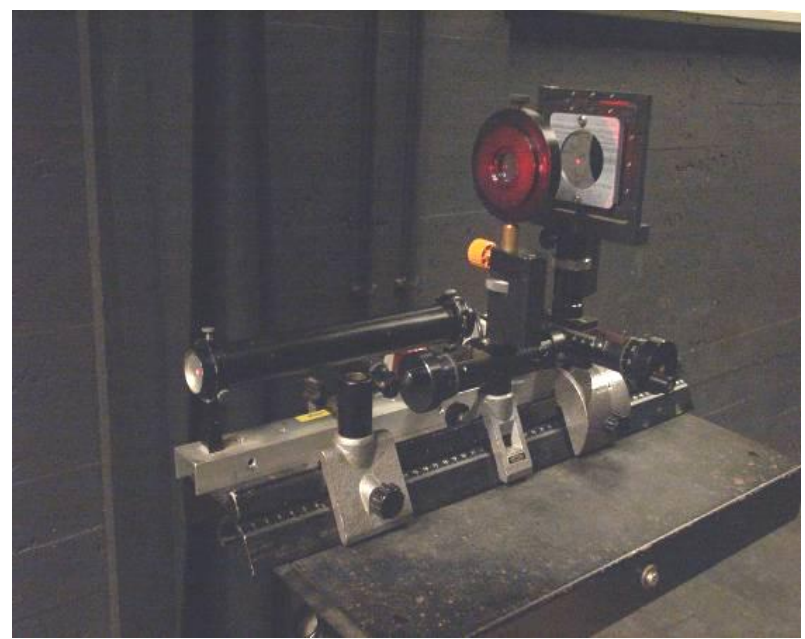

Figure 7: Photo of the sight simulator consisting of a lens and a mirror. The position of the mirror could be adjusted along the optical axis of the lens to simulate a sight with different observation distances.

\subsection{Results and comparison}

For different values of $\varepsilon$ we have taken photos of the intensity distribution of the laser beam and measured both the radius and collected power of the retro-reflected beam in the plane of the detector.

In the top panels of Figure 8 we show photos of the retro-reflected beam for $\varepsilon=-2,-1,0,1$, and $2 \mathrm{~mm}$. These photos have been taken by placing a white sheet of paper against the lens of the detector. Of course, a small hole was made in the paper at the position of the laser so not to block the original laser beam. Although not clearly visible from the photos, circular fringe patterns can be observed. The bottom panels show the corresponding beam shape as calculated by our model. The size of the photographed beams match with the simulated beams and it is observed that the fringe patterns match too. The non-circular features in the in the bottom panel images are due to undersampling in the simulations (Moire).
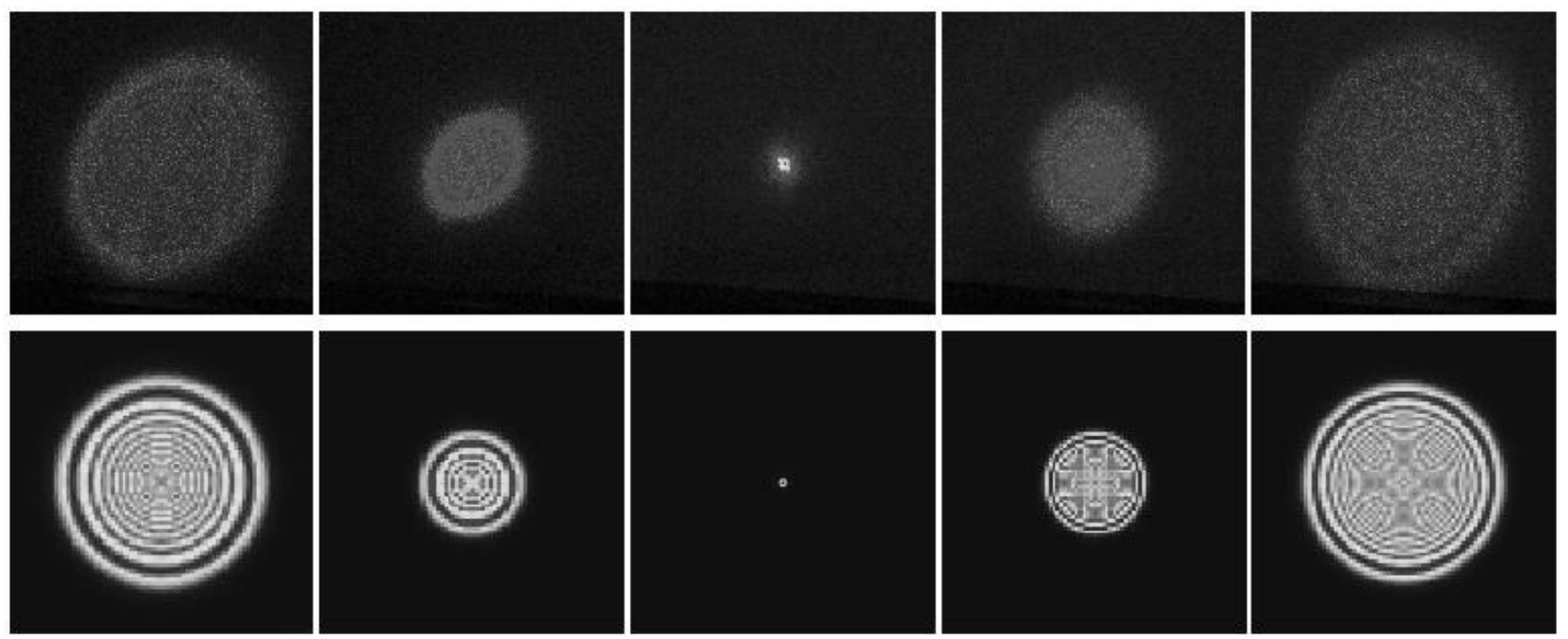

Figure 8: Top panels) Photos of the retro-reflected beam just in front of the detector lens for $\varepsilon=-2,-1,0,1,2 \mathrm{~mm}$ (from left to right). Bottom panels) The beam shape as predicted by our model. 

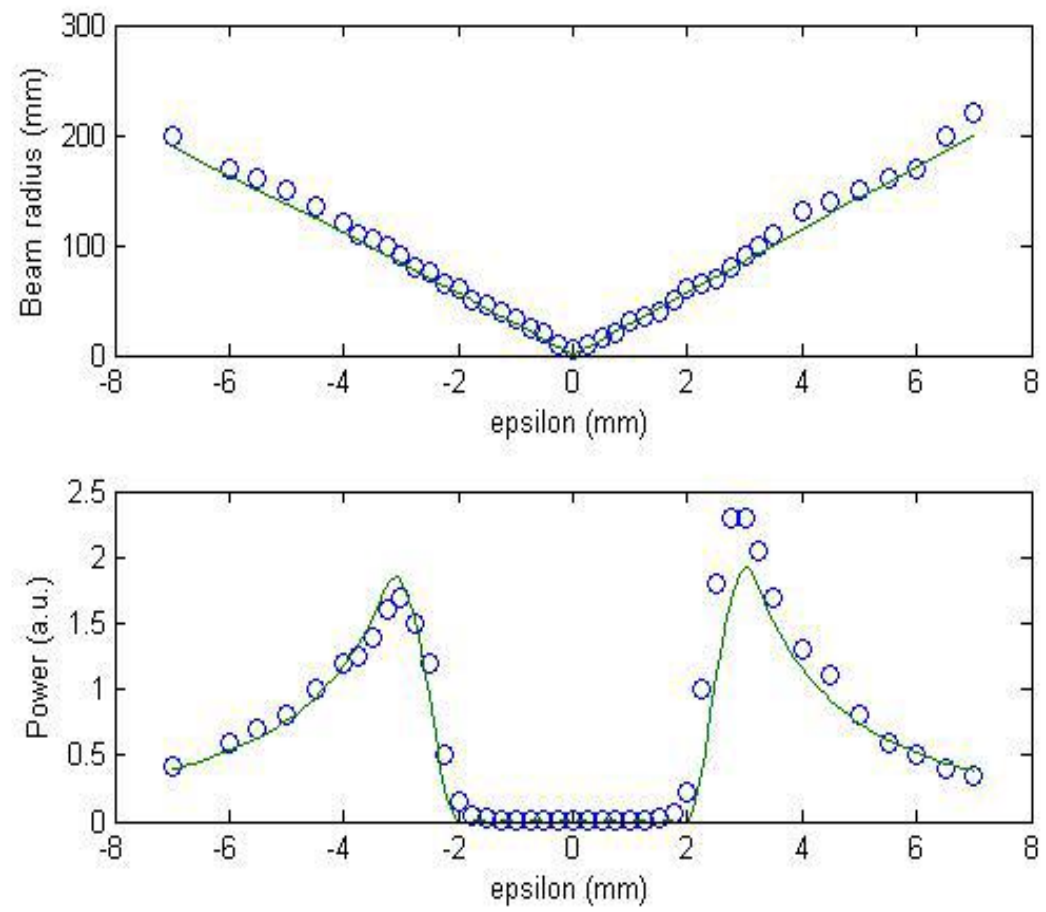

Figure 9: Measurements and prediction of the validation of the defocusing effect.

Besides making photos, the beam size has also been measured with an accuracy of $5 \mathrm{~mm}$. To measure the exact beam size is difficult because it is difficult to estimate where the intensity has decreased below a certain level. In the top panel of Figure 9 we show the results for the beam waist as measured (circles) and as predicted by our tool (solid line). The results obtained with the tool are in agreement with experiment to within $8 \%$. The largest differences are found for large values of $\varepsilon$. For these beam sizes the exact size of the beam are more difficult to measure.

The bottom panel shows the results for the collected power as measured (circles) and as predicted by our tool (solid line). The shape of the power curve initially may seem surprising, but can be explained as follows. When $|\varepsilon|<2 \mathrm{~mm}$, the retro-reflected beam is so small that no power falls upon the lens of the detector. Recall that the detector is placed 74 $\mathrm{mm}$ off-axis. At $|\varepsilon|=2 \mathrm{~mm}$, the size of the retro-reflected beam is $60 \mathrm{~mm}$ (see top panel of Figure 9), which corresponds with the distance between the center of the laser beam and the edge closest to the laser beam. Between $2 \mathrm{~mm}<|\varepsilon|<3$ $\mathrm{mm}$ the power rapidly increases because a larger part of the retro-reflected beam falls upon the lens. At roughly $|\varepsilon|=3$ $\mathrm{mm}$, the maximum power is read. The beam size is $90 \mathrm{~mm}$ and the complete lens is illuminated. For $|\varepsilon|>3 \mathrm{~mm}$, the size of the beam further increases, so that the intensity is spread over a larger area and the total power collected by the lens decreases again.

From the bottom panel of Figure 9 it is also seen that the measured intensity is asymmetric around $\varepsilon=0 \mathrm{~mm}$. One peak is significantly larger than the other. Although a slight asymmetry of several percent is expected, because for negative values of $\varepsilon$ the beam exiting the optical sight has some losses at the edges, it should not be as significant as measured. No explanation is found yet, but it is believed that this is not an error created by the model.

It is concluded that, except for the asymmetry in the measured power the measurement and prediction by our tool are in agreement. In a similar fashion other dependencies, such as tilt and off-axis displacement of the detector, have been validated. 


\section{CONCLUSIONS}

We have investigated the possibilities of optical sight detection based upon the cat's eye effect. To be able to estimate detection distances and the influence of design parameters, a tool has been developed to model the numerous effects that influence the strength of the retro-reflected signal. This paper gives an overview of the effects that have been included in the model and show one of the validation results of this model. Confident that our model correctly predicts these basic optical parameters, we now intend to further develop this model. So far, the emphasis of the tool was on detection. A logical next step is to expand the capabilities to identification of the optical sight. Here one should think of frequency estimation algorithms. In non-imaging seekers, reticles modulate the beam to gain information on the position of its target. This modulation process also modulates the retro-reflected beam which allows for identification of IR seekers.

More general, the development of this tool allows us to make accurate predictions about the possibility to detect optical sights over distances of several kilometers. Furthermore, different detection systems can be modeled to compare their performance, allowing us to further improve the capabilities of this kind of systems.

\section{REFERENCES}

1. L.C. Andrews, "Field guide to atmospheric optics", SPIE Field Guides Vol. FG02 (2004).

2. Gerald C. Holst, "Electro-optical imaging system performance", SPIE optical engineering press ( $2^{\text {nd }}$ ed., 2000) chapter 15 .

3. Akira Ishimaru; Wave Propagation and Scattering in Random Media; Academic Press, 1978. 